Paredes, J. (2017). Planificación financiera ante la perspectiva organizacional en empresas cementeras del estado ZuliaVenezuela. Económicas CUC, 38(1), 105-132. DOI: http:// dx.doi.org/10.17981/econcuc.38.1.05

\title{
Planificación financiera ante la perspectiva organizacional en empresas cementeras del estado Zulia-Venezuela
}

\section{Financial planning before the organizational perspective in cement companies of the Zulia State}

DOI: http://dx.doi.org/10.17981/econcuc.38.1.05

Jeeleen Paredes-Chacín ${ }^{1}$

\section{Resumen}

El análisis de la planificación financiera en empresas cementeras del estado Zulia, Venezuela, fundamenta el objetivo del presente artículo. Desde la perspectiva metodológica, se establece una investigación analítica descriptiva, con diseño no experimental, transeccional, y cuyas bases teóricas se fundamentan en la planificación financiera. La investigación se tipifica como descriptiva con método cualitativo. La selección de la técnica permitió el desarrollo de un cuestionario estructurado cuyos resultados determinan que según la aplicación de la planificación financiera, se determinan los elementos que intervienen en la planificación fundamentada en la perspectiva organizacional, lo cual incide en la elaboración de presupuestos y, a su vez, son determinantes en las decisiones de financiamiento las herramientas de control financiero, lo que permite la comparación de índices financieros y análisis de las desviaciones.

Palabras clave: planificación financiera; perspectiva organizacional; sector cementero.

Recibido: 16/4/2017 Devuelto para revisión: 27/5/17 Aceptado: 2/6/2017.

\footnotetext{
${ }^{1}$ Cursante de estudios Doctorales en Ciencias Sociales. Magister en Gerencia Financiera. Lcda. en Administración Universidad del Zulia. Administradora (E) Vicerrectorado Académico de la Universidad del Zulia. jeeleenparedes@gmail. com
} 


\begin{abstract}
The analysis of the financial planning in cement companies of the Zulia-Venezuela State, bases the objective of this article. From the methodological perspective, a descriptive analytic research, non-experimental, transectional design, and whose theoretical bases are based on financial planning, research is typified as descriptive, qualitative method. The selection of the technique allowed the development of a structured questionnaire whose results determine that according to the application of financial planning, the elements that intervene in the planning based on the organizational perspective are determined, which influences the budgeting and, in turn, are decisive In financial decisions, the tools of financial control that allows the comparison of financial indexes and analysis of the deviations.
\end{abstract}

Keywords: Financial Planning, Organizational Perspective, cement sector.

\title{
Introducción
}

La planificación financiera se constituye como parte de los componentes clave para la gestión efectiva de toda organización, su aplicación contribuye con la administración organizacional impulsada para alcanzar los objetivos previa a la operatividad de las funciones relacionadas que soportan las finanzas corporativas, por lo cual resulta imprescindible la definición de sistemas de evaluación y control que garanticen óptimos resultados financiero-operacional en el contexto organizacional que amerite su aplicación.

En el ámbito mundial, se observan los avances de los procesos financieros orientados hacia consolidar nuevas formas de lograr la competitividad entre perfiles empresariales indistintamente cual sea su sector económico, y el modelo de estructura organizacional 
adaptada, esto implica enfrentarse a un entorno macroeconómico cambiante, lo que influye en todos los sectores de la economía. Por lo que, el desarrollo y direccionamiento estratégico que se impulsa desde las empresas en este caso las referidas al sector cementero del estado Zulia-Venezuela, implica poder superar debilidades relacionadas con la gestión financiera que se proyecta hacia factores externos que resultan determinantes e inciden en la definición del modelo económico, como lo son la escasa o nula identificación de los procedimientos y técnicas que intervienen en el proceso de la planificación financiera, así como la falta de definición e implementación de estrategias que permitan direccionar el comportamiento financiero o ciclo del efectivo, partiendo de indicadores que representa la información utilizada para la toma de decisiones como proceso imprescindible que promueve el éxito organizacional.

En función de lo descrito, se define como objetivo general analizar la planificación financiera en las empresas cementeras del estado Zulia, cuyo posicionamiento alcanzado en el marco del sector producción se ubica en la última década como un sector empresarial de alta importancia para la economía Venezolana. Para la estructura de desarrollo del presente artículo, se estableció el análisis teórico relacionado con la variable planificación financiera cuya estructura de contenido partió de la conceptualización de las dimensiones que sustentan el estudio desde el contexto de las empresas objeto de estudio. Asimismo, se hace referencia sobre la perspectiva de la planificación financiera y su importancia como proceso estratégico empresarial, sus etapas, así como también la vinculación entre planificación estratégica y la financiera para considerar la perspectiva y crecimiento organizacional basado en la planificación financiera cuyo análisis permitió concebir el enfoque metodológico y aspectos concluyentes.

\section{Planificación financiera y su importancia como proceso estratégico empresarial}

El alcance de la planificación financiera ha logrado direccionar la efectiva funcionalidad de procesos administrativos direccionados para garantizar la toma de decisiones basadas en el denominado el plan financiero concebido en el corto o largo plazo. Sin ligar a dudas el resultado de una decisión ejecutiva, amerita sustentarse en función de los avances que en materia de procesos financieros y contables han sido desarrollo de acuerdo con las potencialidades de los recursos humanos, así como de los activos materiales que disponen 
las empresas para desarrollar y consolidar el crecimiento financiero; así como lograr una ampliación de su mercado potencial; como también la ampliación de sus líneas de crecimiento.

En función de lo descrito, se considera lo planteado por Amézquita, P. (2014: 48), quien concibe que la relación de intercambio es el precio del bien, que un país inicialmente exporta dividido por el precio del bien que inicialmente importa, cuando la relación de intercambio del producto exportado cae, el país pierde, lo que da lugar a la posibilidad teórica, planteada por Bhagwati (2004), de que un país se encuentre condiciones débiles después de empezar a exportar que si no hubiese crecido vía exportaciones, fenómeno llamado "crecimiento empobrecedor".

En función de ello, se requiere emprender acciones para aprender a utilizar el poder del Estado para potenciar sus propias capacidades de cara a los mercados nacionales e internacionales, que permita no sólo obtener rentas, como ha sucedido en entornos sociales desfavorables, sino también consolidar estabilidad empresarial, siendo esta la razón por la que el sector cementero busca aplicar de forma integral la planificación financiera, respaldada mediante el uso de las tecnologías de información, así como evaluando de forma progresiva y constantemente las operaciones internas en pro al logro de los objetivos previamente establecidos. La orientación se fundamenta en garantizar la capacidad de solvencia financiera, optimización del efectivo, mejorar la estructura del capital, aminorando los riesgos y cumpliendo de una planificación financiera en mira hacia una gestión efectiva.

De acuerdo con lo expuesto la planificación financiera, contribuye de forma directa a lograr una activa participación en las decisiones orientadas hacia la minimización de costos, controles escritos de calidad, programas de pedidos o despachos, eficiencia administrativa, alta utilización de los recursos, productividad elevada y coordinación adecuada de las actividades administrativas. Asimismo, la conducción financiera, estará presente en las decisiones relacionadasconladiferenciaciónybúsquedademecanismosprevistosparapropiciar la conquista y la fidelización de la clientela. De igual manera, contribuye a estructurar diversas políticas en todos los campos donde esté presente el capital aportado por los inversionistas o suministrado por los demás sectores que apoyan la labor de las organizaciones, por lo que resulta de interés analizar las cinco etapas que fundamentan el proceso de la planificación financiera. 


\section{Etapas de la planificación financiera}

Para lograr la efectividad del proceso de planificación financiera cuyo impacto genera márgenes de estabilidad y confiabilidad de los diferentes sectores empresariales donde se establece se requiere que su concepción sea asumida de forma integral, por lo que se considera su viabilidad en el marco de la estrategia empresarial, sin embargo se quiere para su viabilidad ser estructurada de forma sistémica, por lo cual se plantean las cinco etapas fundamentales para su viabilidad: definición del objetivo, basado en los intereses de la empresa, análisis situación que trasciende la identificación del estado actual de la gestión, para lo cual se utilizan diferentes herramientas gerenciales que permiten identificar oportunidades y amenazas del sector para proceder con la definición de acciones estratégicas para su implementación, sin dejar d destacar la capacidad de concebir la evaluación y el control correspondiente, ver figura 1.

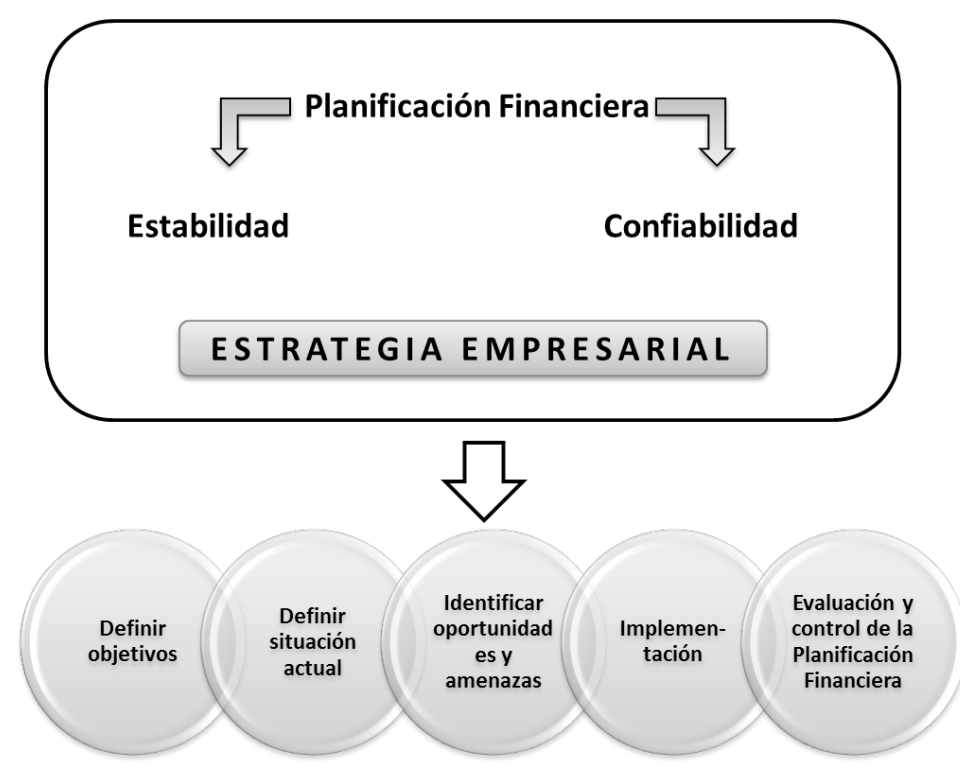

Figura 1. Etapas de la planificación financiera

Fuente: Adaptado de Brealey y Myers, 2010. 
A partir de la fundamentación realizada a cada una de las etapas, se procede a complementar con los principales componentes conceptuales que rigen el referido proceso según se indican a partir de la denominada:

Estrategia financiera: mediante el cual se está inmersa las características fundamentales que la hacen relevante dentro del proceso de planificación estratégica, pues considera los medios que se deben emplear para cumplirlos objetivos de la empresa y llegar al éxito organizacional en un mundo cambiante donde la incertidumbre se hace mayor cada día. Francés (2001) afirma que "la estrategia se refiere a la combinación de medios a emplear para alcanzar los objetivos en presencia de la incertidumbre”. Estas definiciones exponen la estrategia como el conjunto de acciones o guías que van a dirigir los objetivos para alcanzar las metas organizacionales. A través de esta se "potencian los recursos y capacidades empresariales y generar ventajas competitivas sostenibles en el tiempo para una organización”. Cerromatoso, (2014:65).

Se prevé que la definición de estrategias financieras, se llevan a efecto identificando primeramente los aspectos relacionados con las áreas claves en las que se enfoca la gestión y razón de ser de la organización; y donde el director financiero las concibe en el marco de la competitividad basada en procesos regidos por la innovación, la financiación y el riesgo financiero; sin dejar de destacar la definición del objetivo estratégico el cual direcciona la meta a alcanzar. Lo descrito se fundamenta en que "El éxito de la estrategia de innovación dependerá si esta se ajusta a las características internas de la empresa y si sea cómoda a las circunstancias externas del entorno en el que actúa”. Villalba, M., Hurtado, H., Guarín, H. \& Casas, J. (2013: 20).

\section{Vinculación entre planificación estratégica y planificación financiera}

La vinculación entre la planificación estratégica y financiera es determinante para la coherencia y factibilidad del plan que ha de proyectarse en el contexto empresarial. Su clara definición es requerida para verificar los futuros estándares de ingresos, así como los egresos que soportan el ejecútese como tal. Sin lugar a dudas, uno de los componentes esenciales en el desarrollo de la planeación estratégica, es el referido a lo financiero, se constituye en el subsistema mediante el cual se prevé la eficiencia de lo programado en función de las necesidades y objetivos empresariales. 
La planificación estratégica, necesariamente amerita responder a los preceptos básicos que garantizan el direccionamiento organizacional, en consecuencia las etapas de diagnóstico, análisis, reflexión y toma de decisiones colectivas, se constituyen como eje rector que respaldan el quehacer y el camino que deben recorrer en el futuro las organizaciones. Por tratarse de una proyección en el largo plazo, necesariamente tienen que tomar en cuenta los principios de gestión y los objetivos de la empresa. De tal manera, que la planificación financiera por el contrario, es un proceso a corto plazo, esta se inscribe dentro en el marco de la planificación general desde el momento en que se establece la misión y una visión. Definida la "la misión, al sentido claro de propósito o dirección que define las metas organizacionales y los objetivos estratégicos". Cantillo (2013:136).

Sin lugar a dudas, una de las realidades de las organizaciones es que prevalece la importancia de la gestión financiera y sus implicaciones para garantizarla utilización de recursos en función de una apropiada administración. En tal sentido, el manejo integral de elementos de rentabilidad, necesidad de financiamiento, inversión, inflación, tasa de interés y crecimiento, hacen de la planificación financiera un recurso fundamental de la gerencia. En función de ello, se hace referencia a los elementos que soportan la planificación financiera.

\section{Elementos de la planificación financiera}

Desde una perspectiva organizacional, así como para llevar a efecto el proceso de toma de decisiones que se reflejen en una gestión efectiva, se requiere que los miembros del staf ejecutivo de las empresas sean capaz de desarrollar competencias cognitivas y técnicas sobre las perspectivas financieras y por ende ser capaz de generar respuestas acordes ante posibles debilidades, riesgos e incertidumbres que surjan de un contexto regido por efectos de la competitividad empresarial surgida ante entornos globales. En tal sentido, las formas y asertividad en la elección de estrategias amerita responder de forma integral a los escenarios que se presenten. "Desde este punto de vista implica una responsabilidad en torno a la infraestructura de soporte para que se desarrolle la perspectiva individual, en procura de mejorar los procesos, la tecnología, la cultura”. Rodríguez, M. \& González, (2013: 96). 
La conceptualización de la planificación financiera debe estructurarse por una serie de elementos que la soportan, entre ellas: la misión, cuyo fin es determinar en qué consiste el negocio y cuál debería ser, los objetivos, concebidos como un fin concreto generalmente cuantificables y un horizonte temporal hacia los cuales se dirige la actividad, seguido por la definición de estrategias, que respondan al proyecto que conecta a la empresa con el entorno y con el futuro y tiene por finalidad el garantizar la consecuencia de los propósitos de la empresa, las políticas, concebidas como los principios generales para la actuación de los administradores de guiar y orientar el pensamiento así como también la toma de decisiones.

De igual forma, se destacan los procedimientos, que constituyen la guía de acción más que pensamientos y acciones que se requieren para operar sobre problemas estructurados, las normas, como las instrucciones en el uso de una acción, para proceder con los denominados programas, que se sustentan en los planes específicos mediante la cual, se concretan metas, políticas, procedimientos y por último lo referido al presupuesto considerado como la expresión en términos monetarios de un plano programa el cual amerita responder según la perspectiva organizacional.

\section{Perspectiva y crecimiento organizacional basado en la planificación financiera}

Las perspectivas de las organizaciones son proporcionales al crecimiento de las organizaciones, el éxito de estas, tienden al crecimiento y a la ampliación de sus actividades, ya sea en términos de tamaño y recursos o en la expansión de sus mercados o en el volumen de sus operaciones. El crecimiento es una consecuencia inevitable del éxito organizacional, por lo que se establece una competencia más aguda, en la medida que aumenta los mercados y los negocios crecen también los riesgos en la actividad organizacional, principalmente en la actividad empresarial. Para Villalba, M., Hurtado, H., Guarín, H. \& Casas, J. (2013: 18), En un ambiente tan dinámico, turbulento y amenazante, como el que se vive en los negocios, es conveniente fomentar las innovaciones. Algunas organizaciones rentables lo son gracias a su afán de cultivar la innovación en todos los niveles de la organización. 
Asimismo, el grado de compromiso de todo recurso humano que integra la organización, responde al bienestar integral, considerándose como un fin para el buen desempeño en sus funciones. De tal manera, se considera que "un estado de salud óptimo facilita la manifestación del potencial humano para destacarse en un cargo, otorgando así estabilidad a la organización. Por ello individuos saludables generan organizaciones exitosas”. Suarez, Y. \& Mendoza, J. (2014: 138). De igual forma, se define cada uno de los componentes considerados para llevar a efecto la planificación financiera, se destacan:

\section{- Presupuesto Financiero}

Referido a los recursos económicos y financieros necesarios para desarrollar las actividades o procesos y/o para obtener los medios esenciales que deben calcularse, como el costo de la realización, el costo del tiempo y el costo de adquirir nuevos recursos. Comúnmente la factibilidad es la parte más importante, ya que con ella se resuelven otras insuficiencias de otros recursos. Koontz y Weihrich (2004), lo definen como la formulación de resultados esperados expresada en términos numéricos, términos financieros, en términos de horas-hombre o en cualquier otro término numéricamente medible. Es un instrumento utilizado tanto para planear como controlar las actividades financieras futuras y que permite comparar el desempeño real con los pronósticos.

\section{- Fuentes de financiamiento}

Los recursos financieros son los más escasos en la actividad empresarial, razón por la cual, se requiere de toma de decisiones asertivas al momento de evaluar las posibles fuentes de financiamiento de la actividad, en tal caso que los recursos financieros internos son insuficientes las empresas deben recurrir a financiarse a través de recursos financieros a largo plazo, ya sean propios (auto financiación o a través de deudas (terceros), que le permitan obtener los recursos necesarios para seguir produciendo bienes y servicios o para tener mayor capacidad de respuesta a las necesidades financieras presentadas en un tiempo de terminado por la organización. 


\section{- Financiamiento interno}

La auto financiación o financiamiento interno, consiste en la inversión de los recursos generados por la empresa como producto de su actividad económica. Para Mocla (2004), la capacidad de auto financiación de una empresa viene dada por el flujo neto de caja, que se obtiene por la suma del beneficio neto del ejercicio más las amortizaciones. Y como no todo el beneficio forma parte de la autofinanciación, sino sólo aquella parte del mismo, que no distribuye entre los socios. Por lo tanto dependerá de la política de distribución del beneficio que sigue la empresa.

\section{- Financiamiento externo}

Es importante distinguir entre la financiación externa y la financiación ajena (la primera incluye las aportaciones de capital de los propietarios y la segunda no). El proceso de financiación externa lleva implícito dos grandes medios, el endeudamiento con terceros o financiación a través de deuda y la financiación de capital propio. A continuación, se expresan en detalle cada una de ellas.

- El endeudamiento o financiamiento a través de deuda: está representado por las obligaciones de pago que se tienen con terceros, las cuales se adquieren a medida que la empresa pierde capacidad de mantenerse a través de la autofinanciación. Para Santandreu y Santandreu (2000)

- Financiamiento de capital propio: se puede decir que el capital social o propio de la empresa constituye la financiación básica, Santandreu y Santandreu (2000), definen el capital como las aportaciones desembolsadas o comprometidas por los socios y la suma del valor nominal de las acciones de la sociedad.

- Renting o financiamiento por medio de los inventarios: a criterio de Santandreu y Santandreu (2000), es un producto y también un servicio que permite a los usuarios la utilización de unos bienes, en régimen de alquiler a largo plazo que puede oscilar entre dos y cuatro años, más una serie de servicios relacionados con el uso y funcionamiento del mismo. Puede interpretarse como aquel financiamiento donde se utiliza el inventario como garantía de un préstamo y confiere el acreedor un derecho de uso y disfrute a largo plazo. 


\section{Planificación financiera financiamiento en el argo plazo}

El proceso de planificación financiera a largo plazo o estratégicos, según Santandreu y Santandreu (2000), consiste en la elaboración de previsiones a corto, medio y/o largo plazo. En función de esta definición, su horizonte temporal se sitúaentre3o 5años". La planificación financiera intenta analizar el futuro de las empresas, con base de la realización de diferentes escenarios, con la finalidad de preparar las decisiones futuras con el tiempo máximo posible de anticipación. Donde la incertidumbre en el entorno empresarial se hace cada vez más grande, y por tanto los ejecutivos deben esforzarse en desarrollar todas las posibles alternativas de gestión con sus previsiones para tomar decisiones que perduren en el largo plazo y cumplan con los objetivos financieros y estratégicos.

Para que estas decisiones sean las más acertadas posibles, se hace necesario definir condiciones dentro de la planificación financiera a largo plazo, de modo que se utilice con la mayor eficacia dentro del proceso productivo. Santandreu y Santandreu (2000), resume estas condiciones en:

- Se realice en el marco de la planificación estratégica, con base de la cuantificación de unos objetivos y en función de los medios y recursos.

- La estrategia empresarial comentada se contemple en función de la situación del entorno en cada momento.

- Se ciña a los objetivos globales de la empresa, así como de las características de la organización.

- La planificación financiera se realice en función de la influencia del entorno empresarial, y en función de las variables que se deban utilizar en cada momento, como por ejemplo, estimación, demanda, cuota de mercado, crecimiento de los mismos, coste de los créditos, políticas fiscales, entre otros.

- El control de las desviaciones y correcciones, se realice con base a las estimaciones contempladas.

Según lo expuesto, los planes financieros a largo plazo comienzan con el desarrollo de producto y los objetivos de ventas de la empresa, a tal efecto cuando se preparan estos planes no deberían analizar simplemente las consecuencias financieras más probables, también 
se necesita planificar lo inesperado. Es por ello, que la planificación a largo plazo obliga al gerente financiero a considerar los efectos combinados de todas las decisiones de inversión y financiación de la empresa. Así como proyectar a la empresa, concibiendo la ampliación del mercado tal y como lo plantea Millan y otros (2015), para el que operan permite a las empresas de los países signatarios aumentar de escala (es decir, de tamaño en términos de producción) y mejorar su productividad. Es lo que los economistas llaman "aprovechar las economías de escala”. En este nivel de integración puede que existan productos elaborados dentro del área, que incorporen una cierta proporción de insumos producidos por un país no miembro.

\section{Planificación financiera en el corto plazo}

El término capital de trabajo neto suele asociarse con la toma de decisiones financieras a corto plazo, este término no es más que la diferencia entre los activos y pasivos circulantes. A la administración financiera a corto plazo se le denomina a menudo administración del capital de trabajo, ambas denominaciones tienen el mismo significado. Contreras (2009), plantea que la planificación en la última década, dadas las condiciones del entorno, es concebida fundamentalmente de corto plazo. En el período indicado, esta planificación permite determinar los excedentes o déficit de tesorería previsto según los estados financieros proforma (estado de resultados y balances proyectados). Esta cuantificación es la que permite desarrollar estrategias de inversión (en el caso de que se obtengan excedentes) y estrategias de financiamiento (en los casos en se proyecte déficit). Según el autor Contreras (2009), las partidas más importantes en el activo circulante para la planificación financiera deben considerarse las siguientes variables:

- Flujos de efectivo: el presupuesto de flujo de efectivo es una herramienta fundamental en la planificación financiera a corto plazo, ya que este permite registrar las proyecciones de entradas y salidas de efectivo y el resultado estimado de superávit o déficit en los flujos. La mayor parte de las entradas de efectivo provienen de las ventas, mientras que las salidas pueden agruparse en cuatro categorías: pago de cuentas por pagar; gasto de personal, administrativo y otros; inversiones de capital y por ultimo impuestos, intereses y pago de dividendos. El flujo de efectivo nos indica cuanto financiamiento se necesita obtener para hacerle frente a las operaciones planificadas. 
- Ciclo operativo y ciclo de efectivo: en la actividad a corto plazo se presentan una serie de eventos y deducciones que crean flujos de entradas y salidas de efectivo las que están desfasadas porque el pago de efectivo no se realiza al mismo tiempo que se recibe el efectivo y son inciertos porque las ventas y los costos futuros no pueden predecirse con exactitud. El ciclo operativo es el periodo desde que se adquieren los inventarios, se venden y se cobra el efectivo. El ciclo de efectivo, es el periodo desde el momento en que se paga el inventario hasta que se cobra la venta.

- Interpretación del ciclo de efectivo: el ciclo de efectivo depende del ciclo de inventario, de cuentas por cobrar y de cuentas por pagar. Si crecen los ciclos de inventario y cuentas por cobrar, aumenta el ciclo de efectivo y disminuye si la empresa puede diferir el pago de las cuentas por pagar. La mayoría de las empresas tienen un ciclo de efectivo positivo, por lo que requieren financiamiento para los inventarios y para las cuentas por cobrar. Cuanto mayor sea el ciclo de efectivo, más financiamiento se requerirá. Entre más corto sea el ciclo de efectivo, menor será la inversión en inventario y cuentas por cobrar, por consecuencia el total de activo rotara más. El ciclo de conversión de efectivo puede usarse para trazar políticas de inversión en activos circulantes, partiendo de que la política de capital de trabajo se refiere a dos aspectos básicos: definir el nivel apropiado de activos circulantes y la forma en que estos serán financiados.

- Administración de cuentas por pagar: la administración de cuentas por cobrar es otra variable a considerar en la planificación financiera a corto plazo. Esta dada por la política de otorgamiento de crédito a los clientes y el seguimiento al vencimiento de las facturas. También se debe tener en cuenta los descuentos por pronto pago que se pueden conceder, así como las ventas al contado y el interés cargado por el crédito.

\section{Índices financieros.}

Los índices financieros son realmente útiles si se calculan para una serie de años, que permitan determinar promedios y tendencias; y mucho mejor, si los podemos comparar con diferentes empresas del mismo sector. Para Sánchez (2009), las razones o índices financieros, no son más que relaciones que se establecen entre las cifras de los estados 
financieros, para facilitar su análisis e interpretación. A través de ellas, se pueden detectar las tendencias, las variaciones estaciónales, los cambios cíclicos y variaciones irregulares que puedan presentarlas cifras de los estados financieros. Según el citado autor, dependiendo de la orientación que se dé al cálculo de las razones financieras, éstas se pueden dividir o agrupar en cuatro grandes grupos (liquidez, apalancamiento, actividad y rentabilidad).

\section{Metodología}

El enfoque metodológico se rige mediante una investigación de tipo analítica descriptiva, mediante la cual se determinó el comportamiento de la variable planificación financiera; de a cuerdo con Arias (2012), explica que el aspecto descriptivo consiste en la caracterización de un hecho, fenómeno o grupo, con el fin de establecer su estructura o comportamientos”. Mientras para Balestrini (2006), la investigación de tipo descriptiva, permite describir hechos, situaciones o características de objetos, personas o instituciones en estudio.

El diseño de la investigación, según Sabino (2006), se refiere a los pasos, etapas y estrategias que se aplican para el logro de los objetivos planteados. Mientras para Roldan (2007), el diseño de investigación constituye el plan general del investigador para obtener respuestas a sus interrogantes o comprobarla hipótesis de investigación, estableciendo coincidencia con Arias (2012), para quien el diseño de investigación es la estrategia general que adopta el investigador para responder al problema planteado. En el caso objeto de estudio, el diseño de la investigación se cataloga de campo no experimental; de acuerdo a Hernández, Fernández y Baptista (2010), definen un estudio no experimental, cuando no se da una manipulación deliberada de las variables y sólo se observan y analizan los fenómenos en su ambiente natural.

En tanto, es de campo, por cuanto se recoge la información directamente en el lugar donde se producen los hechos, es decir de los encargados de desempeñarla planificación financiera en las empresas que conforman la población. Al respecto Arias (2012), define esta investigación explicando que "consiste en la recolección de datos directamente de los sujetos investigados, o de la realidad donde ocurren los hechos (datos primarios)" (p.28) 
Con respecto de la población, se seleccionó la población constituida por 02 empresa cementeras del Municipio Maracaibo-Edo. Zulia, seleccionando específicamente las unidades de Finanzas y presupuesto de cada una. Para tales efectos, la población total a considerares de 05 sujetos; debido a las características de la población. Esta se considera de tipo finita y accesible, por lo que no se aplicaron criterios de muestreo si no tipo censo. Para Chávez (2007), las poblaciones finitas son aquellas cuyo número es menor de 100.000. Al respecto indica Roldan (2007), una población es finita cuando sus elementos son numerables donde el investigador reconoce a los sujetos a encuestar.

Para la selección de la técnica se seleccionó la encuesta mediante el diseño de un cuestionario para la recolección de datos, estructurado por 32ítemsde selección múltiple, con tres alternativas de respuesta: Siempre (S), a veces (AV), y nunca (N), estilo escala de Likert; la cual de a cuerdo a Chávez(2007), mide actitud eso predisposiciones individuales la puntuación de cada unidad de análisis se obtiene mediante la sumatoria de las respuestas obtenidas encada ítem, los cuales se construyen en función de una serie de ítems que reflejan una actitud positiva o negativa acerca de un estímulo o referente, cuya interpretación se presenta en el cuadro de baremo siguiente: Siempre (S) 3, A veces (AV) 2, Nunca (N) 1

El poder utilizar los puntajes referidos, permitió calcular el coeficiente de confiabilidad, a través de la formula alfa de Cronbach; mediante el paquete estadístico SPSS18.0 para Windows, los índices arrojados por el mismo determinaron el grado de confiabilidad del instrumento. Al respecto, indica Chávez (2007).

\section{Resultados}

Como resultado, del proceso de aplicación del cuestionario, se obtuvo el resultado de un total de A partir de os resultados obtenidos, previa aplicación del cuestionario con 32 ítems, a través del cual se estudiaron las dimensiones e indicadores que se describen a continuación y que se representan estadísticamente. Con respecto a la misión de la empresa, se observa que 100\% de los encuestados de ambas empresas, considera positiva la opción (a); con respecto a las opciones (b y c), estas fueron negativas; la opción (e) obtuvo valoración regular, infiriendo que en una de las empresas, específicamente en la unidad de análisis 1 , ver tabla 1, se encuentra presente en la misión de la empresa. 
Tabla 1

Perspectiva organizacional en las cementeras del Estado Zulia.

\begin{tabular}{|c|c|c|c|c|c|c|c|c|c|c|c|c|c|}
\hline \multirow{3}{*}{ La misión de la empresa: } & \multicolumn{6}{|c|}{$\begin{array}{l}\text { Alternativas de Respuestas } \\
\text { (1) Unidad de análisis }\end{array}$} & \multicolumn{6}{|c|}{$\begin{array}{l}\text { Alternativas de Respuestas } \\
\text { (2) Unidad de análisis }\end{array}$} & \multirow{3}{*}{$\begin{array}{l}\text { Media } \\
\text { del } \\
\text { ítem }\end{array}$} \\
\hline & \multicolumn{2}{|c|}{$\mathbf{S}$} & \multicolumn{2}{|c|}{ AV } & \multicolumn{2}{|c|}{$\mathbf{N}$} & \multicolumn{2}{|c|}{$\mathbf{S}$} & \multicolumn{2}{|c|}{ AV } & \multicolumn{2}{|c|}{$\mathbf{N}$} & \\
\hline & $\mathbf{F C}$ & $\%$ & FC & $\%$ & $\mathbf{F C}$ & $\%$ & FC & $\%$ & FC & $\%$ & $\mathbf{F C}$ & $\%$ & \\
\hline $\begin{array}{l}\text { a) Describe el propósito de } \\
\text { la organización }\end{array}$ & 3 & 100 & 0 & 0 & 0 & 0 & 2 & 100 & 0 & 0 & 0 & 0 & 3,00 \\
\hline $\begin{array}{l}\text { b) Señala el futuro de la } \\
\text { organización }\end{array}$ & 1 & 33,3 & 0 & 0 & 2 & 66,7 & 0 & 0 & 1 & 50 & 1 & 50 & 1,60 \\
\hline $\begin{array}{l}\text { c) Determina en qué } \\
\text { negocios participará y en } \\
\text { cuales no }\end{array}$ & 0 & 0 & 0 & 0 & 3 & 100 & 0 & 0 & 0 & 0 & 2 & 100 & 1,00 \\
\hline $\begin{array}{l}\text { d) Establece los mercados a } \\
\text { los que servirá, }\end{array}$ & 0 & 0 & 0 & 0 & 3 & 100 & 0 & 0 & 0 & 0 & 2 & 100 & 1,00 \\
\hline $\begin{array}{l}\text { e) Indica cómo se } \\
\text { administrará la } \\
\text { compañía }\end{array}$ & 2 & 66,7 & 0 & 0 & 1 & 33,3 & 0 & 0 & 0 & 0 & 2 & 100 & 1,80 \\
\hline f) La razón del negocio & 3 & 100 & 0 & 0 & 0 & 0 & 2 & 100 & 0 & 0 & 0 & 0 & 3,00 \\
\hline $\begin{array}{l}\text { g)La estrecha relación } \\
\text { entre la comunidad y la } \\
\text { organización }\end{array}$ & 2 & 66,7 & 1 & 33,3 & 0 & 0 & 0 & 0 & 0 & 0 & 2 & 100 & 2,20 \\
\hline $\begin{array}{l}\text { h) La aplicación de } \\
\text { adecuadas estrategias de } \\
\text { negocios }\end{array}$ & 3 & 100 & 0 & 0 & 0 & 0 & 0 & 0 & 0 & 0 & 2 & 100 & 2,00 \\
\hline $\begin{array}{l}\text { i) Estudio y delimitación de } \\
\text { mercado }\end{array}$ & 0 & 0 & 0 & 0 & 3 & 100 & 0 & 0 & 0 & 0 & 2 & 100 & 1,00 \\
\hline $\begin{array}{l}\text { j)Las necesidades de los } \\
\text { directivos de la organización }\end{array}$ & 3 & 100 & 0 & 0 & 0 & 0 & 1 & 50 & 0 & 0 & 1 & 50 & 2,60 \\
\hline Total del item & & & & & & & & & & & & & 1,92 \\
\hline
\end{tabular}

Fuente: Elaboración Propia 
Los resultados, aunque fueron de valoración regular reafirman que en la declaración de la misión debe describir el propósito de la empresa, determina en qué negocios participará, la razón de ser; señalar el futuro de la organización y establecer las bases para la toma de decisiones; identificar a la empresa con sus clientes a qué mercados servirá. lo que indica que las empresas cementeras se enfoca principalmente en indicar a donde se desea llegar; en el propósito estratégico; transmitir fuerza y profunda inspiración a la organización; y el planteamiento de retos que justifiquen el esfuerzo; considerando esto como una declaración de intenciones a cerca de la situación deseable en un futuro lejano.

Con respecto, a la dimensión perspectiva organizacional, Cantillo (2013), expone que la utilización del concepto de desempeño y la implementación de sus sistemas de medidas, ha sido uno de los temas de mayor importancia en la administración. Como parte de los resultados se presenta en tabla 2 . Lo referido al estudio de la dimensión.

Sin lugar a dudas, los objetivos de la empresa establecen los parámetros mencionados, ya que los objetivos financieros, son parte del área funcional y se concretan a los aspectos de liquidez, financiamiento, inversión, entre otros; como situaciones financieras a alcanzar cuando más en un año. Los resultados establecen el cumplimiento de los objetivos empresariales con la excepción de los manuales, los cuales no se establecen en las empresas públicas.

Los resultados establecen el cumplimiento de los objetivos empresariales según los postulados de Garrido (2006), el cual indica que las actividades de la planificación se verán influenciada por los recursos disponibles o alcanzables de la organización. De ellos dependerá que se logre o no los objetivos, no obstante el comportamiento de los recursos humanos, financieros y tecnológicos son los que se han considerado como los más importantes para la organización y su eficiente planificación.

Con respecto al presupuesto y flujo de efectivo, son considerados como parte de los elementos más útiles en la planificación financiera, ya que suministra a los usuarios de los estados financieros las bases para evaluar la capacidad que tiene la empresa para generar efectivo y equivalentes al efectivo. Los presupuestos detallados de las diferentes áreas funcionales, de la empresa, partiendo del presupuesto de ventas, de operaciones, de costos operativos, costos de estructura, se llega al presupuesto integrado. Este presupuesto incluye las acciones diarias de la empresa (comprar, vender, cobrar, pagar), ver figura . 
Tabla 2

Perspectiva organizacional

\begin{tabular}{|c|c|c|c|c|c|c|c|c|c|c|c|c|c|}
\hline \multirow{3}{*}{ Los objetivos de la empresa: } & \multicolumn{6}{|c|}{$\begin{array}{c}\text { Alternativas de Respuestas } \\
\text { CATATUMBO }\end{array}$} & \multicolumn{6}{|c|}{$\begin{array}{c}\text { Alternativas de } \\
\text { Respuestas } \\
\text { CEMEX }\end{array}$} & \multirow{3}{*}{$\begin{array}{c}\text { Media } \\
\text { del } \\
\text { ítem }\end{array}$} \\
\hline & \multicolumn{2}{|c|}{$\mathrm{S}$} & \multicolumn{2}{|c|}{ AV } & \multicolumn{2}{|l|}{$\mathbf{N}$} & \multicolumn{2}{|c|}{$\mathrm{S}$} & \multicolumn{2}{|c|}{ AV } & \multicolumn{2}{|c|}{$\mathbf{N}$} & \\
\hline & FC & $\%$ & FC & $\%$ & FC & $\%$ & FC & $\%$ & FC & $\%$ & FC & $\%$ & \\
\hline $\begin{array}{l}\text { a) Se establece para ser } \\
\text { alcanzados en un período } \\
\text { determinado }\end{array}$ & 3 & 100 & 0 & 0 & 0 & 0 & 2 & 100 & 0 & 0 & 0 & 0 & 3,00 \\
\hline $\begin{array}{l}\text { b) Se concretan } \\
\text { principalmente en los } \\
\text { aspectos de financiamiento }\end{array}$ & 0 & 0 & 1 & 33,3 & 2 & 66,7 & 0 & 0 & 0 & 0 & 2 & 100 & 1,20 \\
\hline c) Se revisan anualmente & 3 & 100 & 0 & 0 & 0 & 0 & 2 & 100 & 0 & 0 & 0 & 0 & 3,00 \\
\hline $\begin{array}{l}\text { d) Establecen situaciones } \\
\text { financieras a alcanzar cuando } \\
\text { más en un año }\end{array}$ & 3 & 100 & 0 & 0 & 0 & 0 & 1 & 50 & 1 & 50 & 0 & 0 & 2,80 \\
\hline $\begin{array}{l}\text { e) Se evalúan a través de } \\
\text { los indicadores de medición, } \\
\text { controlando su cumplimiento }\end{array}$ & 3 & 100 & 0 & 0 & 0 & 0 & 2 & 100 & 0 & 0 & 0 & 0 & 3,00 \\
\hline $\begin{array}{l}\text { f)Los factores externos a la } \\
\text { organización }\end{array}$ & 3 & 100 & 0 & 0 & 0 & 0 & 2 & 100 & 0 & 0 & 0 & 0 & 3,00 \\
\hline $\begin{array}{l}\text { g) El recurso financiero con } \\
\text { el que cuenta la organización }\end{array}$ & 3 & 100 & 0 & 0 & 0 & 0 & 2 & 100 & 0 & 0 & 0 & 0 & 3,00 \\
\hline $\begin{array}{l}\text { h) La adecuada Planificación } \\
\text { Financiera }\end{array}$ & 3 & 100 & 0 & 0 & 0 & 0 & 2 & 100 & 0 & 0 & 0 & 0 & 3,00 \\
\hline $\begin{array}{l}\text { i) La flexibilidad ante } \\
\text { los cambios del entorno } \\
\text { organizacional }\end{array}$ & 3 & 100 & 0 & 0 & 0 & 0 & 2 & 100 & 0 & 0 & 0 & 0 & 3,00 \\
\hline $\begin{array}{l}\text { j) La identificación del } \\
\text { empleado con la organización }\end{array}$ & 1 & 33,3 & 1 & 33,3 & 1 & 33,3 & 2 & 100 & 0 & 0 & 0 & 0 & 1,60 \\
\hline Total del ítem & & & & & & & & & & & & & 2,66 \\
\hline
\end{tabular}

Fuente: Elaboración Propia (2012) 
Tabla 3

Presupuesto y flujo de efectivo

\begin{tabular}{|c|c|c|c|c|c|c|c|c|c|c|c|c|c|}
\hline \multirow{3}{*}{$\begin{array}{l}\text { Los presupuestos y flujo de } \\
\text { efectivo: }\end{array}$} & \multicolumn{6}{|c|}{$\begin{array}{c}\text { Alternativas de Respuestas } \\
\text { CATATUMBO }\end{array}$} & \multicolumn{6}{|c|}{$\begin{array}{c}\text { Alternativas de Respuestas } \\
\text { CEMEX }\end{array}$} & \multirow{3}{*}{$\begin{array}{l}\text { Medic } \\
\text { del } \\
\text { item }\end{array}$} \\
\hline & \multicolumn{2}{|c|}{$\mathbf{S}$} & \multicolumn{2}{|c|}{ AV } & \multicolumn{2}{|c|}{$\mathbf{N}$} & \multicolumn{2}{|c|}{$\mathbf{S}$} & \multicolumn{2}{|c|}{$\mathrm{AV}$} & \multicolumn{2}{|c|}{$\mathbf{N}$} & \\
\hline & FC & $\%$ & FC & $\%$ & FC & $\%$ & FC & $\%$ & FC & $\%$ & FC & $\%$ & \\
\hline $\begin{array}{l}\text { a) Elabora presupuestos } \\
\text { detallados }\end{array}$ & 3 & 100 & 0 & 0 & 0 & 0 & 2 & 100 & 0 & 0 & 0 & 0 & 3,00 \\
\hline $\begin{array}{l}\text { b) Los presupuestos } \\
\text { recogen la planificación } \\
\text { de las diferentes áreas } \\
\text { funcionales }\end{array}$ & 3 & 100 & 0 & 0 & 0 & 0 & 2 & 100 & 0 & 0 & 0 & 0 & 3,00 \\
\hline $\begin{array}{l}\text { c) Elabora presupuestos } \\
\text { integrados }\end{array}$ & 3 & 100 & 0 & 0 & 0 & 0 & 2 & 100 & 0 & 0 & 0 & 0 & 3,00 \\
\hline $\begin{array}{l}\text { d) El reporte de flujo de } \\
\text { efectivo se solicita y analiza } \\
\text { mensualmente }\end{array}$ & 3 & 100 & 0 & 0 & 0 & 0 & 2 & 100 & 0 & 0 & 0 & 0 & 3,00 \\
\hline $\begin{array}{l}\text { e) Los presupuestos } \\
\text { consideran las actividades } \\
\text { financieras diarias de la } \\
\text { empresa }\end{array}$ & 3 & 100 & 0 & 0 & 0 & 0 & 0 & 0 & 0 & 0 & 2 & 100 & 2,20 \\
\hline $\begin{array}{l}\text { f). Fluctuaciones de tipo } \\
\text { cambiario. }\end{array}$ & 3 & 100 & 0 & 0 & 0 & 0 & 1 & 50 & 1 & 50 & 0 & 0 & 2,80 \\
\hline g).Gasto gubernamental & 0 & 0 & 0 & 0 & 3 & 100 & 0 & 0 & 0 & 0 & 2 & 100 & 1,00 \\
\hline h).Estabilidad social & 0 & 0 & 0 & 0 & 3 & 100 & 2 & 100 & 0 & 0 & 0 & 0 & 1,80 \\
\hline $\begin{array}{l}\text { i).Operaciones con bajo nivel } \\
\text { de utilidad }\end{array}$ & 3 & 100 & 0 & 0 & 0 & 0 & 2 & 100 & 0 & 0 & 0 & 0 & 3,00 \\
\hline $\begin{array}{l}\text { j).Carencia de planes } \\
\text { estratégicos }\end{array}$ & 3 & 100 & 0 & 0 & 0 & 0 & 2 & 100 & 0 & 0 & 0 & 0 & 3,00 \\
\hline Total del ítem & & & & & & & & & & & & & 2,58 \\
\hline
\end{tabular}

Fuente: Elaboración propia 
Los riesgos del presupuesto para operaciones internacionales, de acuerdo con los encuestados tanto en el sector público como en el privado, resultaron como principales riesgos las opciones (f, i y j); para ambas empresas "nunca" ocasiona riesgo la opción (g); igualmente la opción (h) fue considerada un riesgo por parte de la empresa Cemex mientras en la empresa Cementos Catatumbo no.

En síntesis los principales riesgos, se encuentran en las fluctuaciones cambiarias, baja utilidad y falta de planeación estratégica. Es importante considerar, los factores externos en la organización para la elaboración de los presupuestos, en caso de la empresa pública la cual se generan presupuestos rígidos, limitando las acciones de aplicación de estrategias financieras.

Tabla 4

Planificación financiera

\begin{tabular}{|c|c|c|c|c|c|c|c|c|c|c|c|c|c|}
\hline \multirow{3}{*}{$\begin{array}{l}\text { Las decisiones de } \\
\text { inversiones a largo } \\
\text { plazo, la empresa: }\end{array}$} & \multicolumn{6}{|c|}{$\begin{array}{c}\text { Alternativas de Respuestas } \\
\text { CATATUMBO }\end{array}$} & \multicolumn{6}{|c|}{$\begin{array}{c}\text { Alternativas de Respuestas } \\
\text { CEMEX }\end{array}$} & \multirow{3}{*}{$\begin{array}{l}\text { Media } \\
\text { del } \\
\text { ítem }\end{array}$} \\
\hline & \multicolumn{2}{|c|}{$\mathrm{S}$} & \multicolumn{2}{|c|}{ AV } & \multicolumn{2}{|c|}{$\mathrm{N}$} & \multicolumn{2}{|c|}{$\mathbf{S}$} & \multicolumn{2}{|c|}{ AV } & \multicolumn{2}{|c|}{$\mathbf{N}$} & \\
\hline & FC & $\%$ & FC & $\%$ & FC & $\%$ & FC & $\%$ & FC & $\%$ & FC & $\%$ & \\
\hline $\begin{array}{l}\text { a) Analiza los } \\
\text { proyectos para } \\
\text { decidir cuáles son } \\
\text { las inversiones } \\
\text { aceptables. }\end{array}$ & 3 & 100 & 0 & 0 & 0 & 0 & 2 & 100 & 0 & 0 & 0 & 0 & 3,00 \\
\hline $\begin{array}{l}\text { b) Considera el } \\
\text { total de recursos } \\
\text { económicos necesarios } \\
\text { para llevar a cabo un } \\
\text { Proyecto de Inversión. }\end{array}$ & 3 & 100 & 0 & 0 & 0 & 0 & 2 & 100 & 0 & 0 & 0 & 0 & 3,00 \\
\hline $\begin{array}{l}\text { c) Determina las } \\
\text { probables fuentes de } \\
\text { financiamiento }\end{array}$ & 3 & 100 & 0 & 0 & 0 & 0 & 0 & 0 & 0 & 0 & 2 & 100 & 2,20 \\
\hline $\begin{array}{l}\text { d) Establece planes } \\
\text { financieros a largo } \\
\text { plazo con el desarrollo } \\
\text { del producto }\end{array}$ & 3 & 100 & 0 & 0 & 0 & 0 & 2 & 100 & 0 & 0 & 0 & 0 & 3,00 \\
\hline
\end{tabular}




\begin{tabular}{|c|c|c|c|c|c|c|c|c|c|c|c|c|c|}
\hline $\begin{array}{l}\text { e) Establece planes } \\
\text { financieros a través } \\
\text { de los objetivos de } \\
\text { ventas de la empresa }\end{array}$ & 3 & 100 & 0 & 0 & 0 & 0 & 2 & 100 & 0 & 0 & 0 & 0 & 3,00 \\
\hline $\begin{array}{l}\text { f).Los objetivos } \\
\text { específicos para cada } \\
\text { variable financiera de } \\
\text { largo plazo }\end{array}$ & 1 & 33,3 & 0 & 0 & 2 & 66,7 & 0 & 0 & 0 & 0 & 2 & 100 & 1,40 \\
\hline $\begin{array}{l}\text { g).La utilización } \\
\text { de métodos para } \\
\text { analizar los proyectos } \\
\text { de inversión partiendo } \\
\text { de criterios estáticos } \\
\text { (Cash-flow estático, } \\
\text { Pay-back o plazo de } \\
\text { recuperación y tasa } \\
\text { de rendimiento), } \\
\text { criterios dinámicos } \\
\text { (VAN, TIR) }\end{array}$ & 0 & 0 & 0 & 0 & 3 & 100 & 0 & 0 & 0 & 0 & 2 & 100 & 1,00 \\
\hline $\begin{array}{l}\text { h).Liquidez que } \\
\text { genera el proyecto }\end{array}$ & 2 & 66,7 & 1 & 33,3 & 0 & 0 & 2 & 100 & 0 & 0 & 0 & 0 & 2,80 \\
\hline $\begin{array}{l}\text { i)Riesgo de la } \\
\text { inversión }\end{array}$ & 2 & 66,7 & 1 & 33,3 & 0 & 0 & 2 & 100 & 0 & 0 & 0 & 0 & 2,80 \\
\hline Total del ítem & & & & & & & & & & & & & 2,63 \\
\hline
\end{tabular}

Fuente: Elaboración Propia (2012)

En cuanto a la inversión a corto plazo, intenta asegurar que la empresa, en su conjunto, y en particular cada uno de los diferentes departamentos que la integran, tomen decisiones de financiamiento e inversión lo más asertivas posibles de modo que logren sus objetivos con eficiencia y eficacia.

Con referencia a las inversiones a largo plazo realizadas por las empresas, se evidencia de acuerdo al cuadro precedente diversidad de criterios, en cuanto a la razón a la cual obedecen las mismas, resultando positivas las opciones (h, i). En tanto resultaron negativas en ambas empresas, las opciones (f y g), aspectos que se contrapone a los postulados de diversos autores tales como Grinblatt y Titman (2003), Besley y Brigham (2000). 
La media del ítem fue de 2,63 de valoración alta por tanto se establece cumplimiento de los procedimientos necesarios para determinar las inversiones a realizar a largo plazo en ambas empresas cementeras; con fundamento en los criterios de Bodie y Merton (1999), "los proyectos comienzan con una idea para acrecentar la riqueza de los accionistas produciendo un nuevo bien o mejorando la forma en que se produce uno ya existente".

Para quien el financiamiento a corto plazo, se está operando con instrumentos que complementan la tradicional línea de crédito o los préstamos de corto plazo o los créditos de corto plazo con garantía de inventarios, el éxito de estas decisiones va a estar definido por la composición de los recursos permanentes, de modo que se optimicen los objetivos estratégicos y financieros de la entidad. El objetivo, es conocer todas las variantes que existen en su entorno macroeconómico para obtener un financiamiento y elegir la que más se adapte a las necesidades y expectativas de las organizaciones estudiadas.

Los objetivos planificados deben estar reflejados notoriamente de forma cuantitativa y cualitativa, contar con un personal calificado y mantener instrumentos de medida de control, donde la comparación de índices involucra los métodos de cálculo e interpretación de índices financieros que presentados oportunamente evidencian y se puede emitir un diagnostico situacional, con el fin de evaluar el desempeño y posición de la empresa, definiendo estrategias para la planificación financiera.

Como se observa en la tabla 5, en ambas empresas resultaron positivas las opciones (a, b, c y d); con respecto a la alternativa (e) esta se emplea solo en la empresa Cementos Catatumbo.

Para constatar que el análisis de las desviaciones en las empresas cementeras estudiadas contribuye positivamente con los aspectos relacionados en las alternativas presentadas el control de las desviaciones y correcciones, se realiza en función de las estimaciones contempladas. En cuanto a la planificación de los correctivos debe realizarse el análisis de las causas de las desviaciones y posterior propuestas de acciones correctivas, donde la comparación relacionadas con las magnitudes financieras de la empresa consiste en comparar los índices de gestión reales con los estimados o planificados, de manera que se puedan detectar a tiempo las desviaciones y realizar el replanteamiento de los objetivos o de las estrategias para cumplir con las metas propuestas. 
Tabla 5

Herramientas del control financiero

\begin{tabular}{|c|c|c|c|c|c|c|c|c|c|c|c|c|c|}
\hline \multirow{3}{*}{$\begin{array}{l}\text { La empresa emplea como } \\
\text { herramienta del control } \\
\text { financiero la comparación } \\
\text { de índices financieros en los } \\
\text { que: }\end{array}$} & \multicolumn{6}{|c|}{$\begin{array}{c}\text { Alternativas de Respuestas } \\
\text { CATATUMBO }\end{array}$} & \multicolumn{6}{|c|}{$\begin{array}{c}\text { Alternativas de Respuestas } \\
\text { CEMEX }\end{array}$} & \multirow{3}{*}{$\begin{array}{l}\text { Media } \\
\text { del } \\
\text { ítem }\end{array}$} \\
\hline & \multicolumn{2}{|c|}{$\mathrm{S}$} & \multicolumn{2}{|c|}{$\mathrm{AV}$} & \multicolumn{2}{|c|}{$\mathbf{N}$} & \multicolumn{2}{|c|}{$\mathrm{S}$} & \multicolumn{2}{|c|}{$\mathrm{AV}$} & \multicolumn{2}{|c|}{$\mathbf{N}$} & \\
\hline & FC & $\%$ & FC & $\%$ & FC & $\%$ & FC & $\%$ & FC & $\%$ & FC & $\%$ & \\
\hline $\begin{array}{l}\text { a) Establecen criterios de } \\
\text { medición en la ejecución } \\
\text { real de los planes. }\end{array}$ & 3 & 100 & 0 & 0 & 0 & 0 & 2 & 100 & 0 & 0 & 0 & 0 & 3,00 \\
\hline $\begin{array}{l}\text { b) Determinan las áreas } \\
\text { críticas de las actividades de } \\
\text { la empresa }\end{array}$ & 3 & 100 & 0 & 0 & 0 & 0 & 2 & 100 & 0 & 0 & 0 & 0 & 3,00 \\
\hline $\begin{array}{l}\text { c) Miden los resultados } \\
\text { arrojados por la ejecución en } \\
\text { un momento determinado }\end{array}$ & 3 & 100 & 0 & 0 & 0 & 0 & 2 & 100 & 0 & 0 & 0 & 0 & 3,00 \\
\hline $\begin{array}{l}\text { d) Se estiman los índices } \\
\text { financieros para una } \\
\text { periodo específico }\end{array}$ & 3 & 100 & 0 & 0 & 0 & 0 & 2 & 100 & 0 & 0 & 0 & 0 & 3,00 \\
\hline $\begin{array}{l}\text { e) Realizar auditorías } \\
\text { y procedimientos } \\
\text { automatizados. }\end{array}$ & 3 & 100 & 0 & 0 & 0 & 0 & 0 & 0 & 0 & 0 & 2 & 100 & 2,20 \\
\hline $\begin{array}{l}\text { f). Se revisan cada } 6 \\
\text { meses el resultado de las } \\
\text { inversiones. }\end{array}$ & 3 & 100 & 0 & 0 & 0 & 0 & 2 & 100 & 0 & 0 & 0 & 0 & 3,00 \\
\hline $\begin{array}{l}\text { g). Se monitorean los } \\
\text { indicadores planificados, al } \\
\text { menos mensualmente. }\end{array}$ & 3 & 100 & 0 & 0 & 0 & 0 & 2 & 100 & 0 & 0 & 0 & 0 & 3,00 \\
\hline $\begin{array}{l}\text { h). Se verifican anualmente } \\
\text { los resultados }\end{array}$ & 3 & 100 & 0 & 0 & 0 & 0 & 0 & 0 & 0 & 0 & 2 & 100 & 2,20 \\
\hline $\begin{array}{l}\text { i). Se miden expresamente } \\
\text { los indicadores financieros }\end{array}$ & 0 & 0 & 0 & 0 & 3 & 100 & 0 & 0 & 0 & 0 & 2 & 100 & 1,00 \\
\hline Total del ítem & & & & & & & & & & & & & 2,57 \\
\hline
\end{tabular}

Fuente: elaboración propia 
De acuerdo con el análisis financiero - estadístico, son tomadas las decisiones para aplicar técnicas y procedimientos que permitan obtener los mejores resultados y evaluar el status financiero de la empresa, detectar cualquier obstáculo existente, estructurar e implementar acciones correctivas.

El proceso puede determinar acciones futuras ante hechos irregulares o en el mejor de los casos podrían tomar previsiones para estar en la capacidad de evitar en lo posible la persistencia de estas situaciones que desvíen el plan original.

La necesidad de la información financiera hace que se produzcan los estados financieros para expresar cuál es la situación financiera, resultado de operaciones y cambios en la situación financiera de una empresa, lo que sirve para la formulación de sus conclusiones sobre el desempeño financiero de la entidad. Por medio de esta información y otros elementos de juicio el usuario general podrá evaluar el futuro de la empresa y tomar decisiones de carácter económico sobre la misma.

\section{Conclusiones}

El analizar la planificación financiera en empresas cementeras del Estado Zulia-Venezuela, permitió concluir que prevalece una débil concepción sobre la gestión integral de la planificación financiera, en ella se concibe el proceso presupuestal atribuida como una herramienta administrativa útil para todas las fases de las operaciones en sector empresarial. No sólo los presupuestos hacen que la administración se anticipe a los problemas que se avecinan, también sirven como normas para el desempeño en tanto avanza la industria, sin embargo, se evidenció una desarticulación entre los procesos que respaldan la planeación financiera en consecuencia se dificulta determinar en qué medida se logra la eficiencia de los procedimientos que respaldan la accesibilidad a fuentes de financiamiento y como se da el seguimiento, evaluación y control del referido proceso.

Por lo que la formulación de presupuestos a largo plazo de las necesidades de financiamiento o financieras es de importancia para el éxito de cualquier organización comercial, está inmersa en la Planificación Financiera de la empresa. En la operatividad financiera, desarrollar la habilidad para pronosticar las ventas es crucial en el esfuerzo para determinar las futuras necesidades financieras. A medida que las ventas aumentan, es necesaria una 
mayor inversión en activos para apoyar este aumento. Este incremento de activos debe ser financiado con cierta combinación de fondos a corto y a largo plazo.

Asimismo, en el marco de la generación de aportes que fundamenten la importancia de la investigación se concibe como estrategias de interés empresarial, la consolidación de sistemas de información que permitan optimar y garantizar el necesario control financiero empresarial. En tal sentido, se confirma que se concreta en la comparación de índices fi- $\square$ nancieros con resultados esperados a través de este proceso se concreta, lo cual permite establecer criterios de medición en la ejecución real de los planes así como determinan las áreas críticas de las actividades de la empresa, además de medir los resultados y estimar los índices financieros para una periodo específico.

Por su parte el análisis de las desviaciones, a través de métodos de control detecta las causas de las diferencias entre objetivos - resultado. Por tanto, la aplicación de correctivos se concretan ante la presencia de desviaciones precisadas tras la aplicación de esta herramienta implícita en la planificación financiera, así como el uso eficaz y eficiente de los sistemas de información (estadísticos y/o contables) lo que permite comparación de las magnitudes financieras de la empresa con los objetivos del plan financiero para el alcance de los objetivos.

Desde la perspectiva de limitaciones presentadas en el desarrollo de la investigación se determinó que la falta de cultura ante la gestión financiera, obstaculizó y afectó el cronograma programado para lograr el objetivo, así como también

En función de lo descrito, se insta a consolidar una nueva visión sustentada en nuevas investigaciones sobre la relevancia de la planificación financiera en el contexto de sectores empresariales orientados, no sólo hacia la competitividad en mercados locales y globales, sino también, a generar la denominada transparencia de gestión basada en el análisis, evaluación, control y comparación de índices financieros como procesos estratégicos para la eficiencia de la gestión financiera.

\section{Referencias}

Amézquita, P. (2014). Minería y Petróleo en Colombia: Maldición interna de los recursos, Revista Económicas CUC, (1), 45-49. 
Arias, F. (2012), El proyecto de investigación. Introducción a la metodología científica. Sexta Edición. McGraw-Hill. México.

Asree, S., Zain, M. y Rizal, M. (2010). Influence of leadership competency and organizational culture on responsiveness and performance of firms. International Journal of Contemporary Hospitality Management, 2(4). 500- 516

Bhagwati, J. (2004). In Defense of Globalization. Oxford University Press.

Brealey, R. A., y Myers, S. C. (2010) Principios de Finanzas Corporativas. McGraw-Hill. México

Besley, S. y Brigman, E. (2001). Fundamentos de administración financiera. 12 ed. McGraw-Hill. México

Burbano, J. y Ortiz, A. (1995). Presupuestos. Enfoque moderno de planeación y control de recursos. 2 ed. McGraw-Hill. Colombia.

Calderón, G., Álvarez, C. y Naranjo, J. (2010) Estrategia competitiva y desempeño organizacional en empresas industriales Colombianas. Innovar, 20 (38). 13-26.

Cantillo, J. (2013). Incidencia de la cultura organizacional en el desempeño. Revista Económicas CUC, 34 (1). 131-152.

Contreras, E. (2009). La Importancia de la Gestión Financiera en la Creación de Valor. 4 ed. McGraw Hill. México.

Corporación Civil Bolsa de Valores de Guayaquil. (1998). Diccionario de Economía y Finanzas. 1 Ed. Guayaquil: Editorial Poligráfica,.

Esteo, F. (1989). Análisis de estados financieros, planificación y control (Comentarios y casos prácticos). Madrid: Centro de Estudios Financieros.

Francés, A. (2001). Estrategia para la empresa en América Latina. Caracas: Ediciones IESA.

Garrido, S. (2006). Dirección Estratégica. 2 Ed. España: McGraw- Hill/Interamericana de España, S.A.U.

González, J., Moreno, L. y Rodríguez, M. (2015). La integración y sus convenios internacionales en America Latina. Económicas CUC, 36(2), 79-94. 
Hernández, R., Fernández, . y Baptista, P. (2010). Metodología de la investigación. 5 ed. México: McGraw-Hill Interamericana.

Koontz, H. y Weihrich, H. (2004). Administración: una perspectiva global. $12^{a}$ ed. México: MX: McGraw-Hill.

Martínez, D. y Milla, A. (2007). Cuadro de mando integral. La elaboración del plan estratégico y su implantación a través del cuadro de mando integral. España: Ediciones Díaz de Santos.

Martínez, M. (2010) Relaciones entre cultura y desempeño organizacional en una muestra de empresas colombianas: reflexiones sobre la utilización del modelo de Denison. Cuaderno de Administración Bogotá (Colombia), 23 (40): 163-190.

Mish, F. (1996). Merriam-Webster's Collegiate Dictionary Tenth ed Inc.. U.S.A.: Frederick C. Mish.

Murugan, M. (2009). A Study on Organizational Culture and Its Impact on the performance of IT Employees in Chennai. The IUP Journal of Management Research. 8 (5). 7-16.

Naranjo, J., Jiménez, D. y Sanz, R. (2011). ¿Es la cultura organizativa un determinante de la innovación en la empresa? Cuadernos de Economía y Dirección de la Empresa. 15. $1-10$.

Quesada, F.J. (1998). Presentación de la información anual. Albacete: Ed. Tébar Flores.

Rodríguez, M. y González, J. (2013). Gestión del Conocimiento y Capital Intelectual, a través de modelos universitarios. Revista Económicas CUC, 34 (1). 85-116.

Rondeau, K. y Wagar, T. (1998). Hospital Chief Executive Officer Perceptions of Organizational Culture and Performance. Hospital Topics, 76(2). 14-21.

Rosenberg, J. (1999). Diccionario de Administración y Finanzas. Barcelona: Océano/Centrum.

Santandreu, E. y Santandreu, P. (2000). Manual de Finanzas. España: Gestión.

Stoner, J. y Freeman, E. (1999). Administración de empresa. Mexico: Prentice Hall.

Suarez, Y. y Mendoza, J. (2014). Indicadores de salud mental y engagement en empleados de unaempresa promotora del desarrollo económico, Económicas CUC, 35 (2), 133-146. 
Terry, G. (1971). Principios de administración. México: CECSA.

Villalba, M., Hurtado, H., Guarín, H. y Casas, J. (2013). Innovación en pymes artesanales de Morroa, Sucre, 2012. Revista Económicas CUC, 34 (1). 15-28.

Wali, S. y Boujelbene Y. (2011). Cultural Influences On TQM Implementation And Financial Performance In Tunisian Firms. Journal Ekonomika A Management. 3. 30-45.

Weston, T. (2006). Fundamentos de Administración Financiera. Vol II y III, La Habana: Editorial Félix Varela. 